BNL-NT-08/13

YITP-SB-08-15

\title{
Threshold Resummation for the Top Quark Charge Asymmetry
}

\author{
Leandro G. Almeida ${ }^{a}$, George Sterman ${ }^{a}$, Werner Vogelsang ${ }^{b}$ \\ ${ }^{a}$ C.N. Yang Institute for Theoretical Physics, Stony Brook University, \\ Stony Brook, New York 11794 - 3840, U.S.A. \\ ${ }^{b}$ Physics Department, Brookhaven National Laboratory, Upton, NY 11973, U.S.A.
}

\begin{abstract}
We study the QCD charge asymmetry in $t \bar{t}$ production at the Tevatron. We investigate the role of higher orders in perturbation theory by considering the resummation of potentially large logarithmic corrections that arise near partonic threshold. This requires us to employ the rapiditydependent anomalous dimension matrices that describe color mixing due to soft gluon emission in both quark- and gluon-initiated processes. The charge asymmetry appears directly in the resummed cross section at next-to-leading logarithm (NLL), and we find that the first-order expansion of the NLL resummed charge asymmetry reproduces the known fixed-order result for the asymmetry well. Beyond its lowest order, the asymmetric component of the cross section is enhanced by the same leading-logarithmic threshold corrections as the total cross section. As a result, the charge asymmetry is robust with respect to the higher-order perturbative corrections generated by threshold resummation. We observe that the asymmetry increases with pair mass and with scattering angle.
\end{abstract}




\section{Introduction}

Heavy quark pair production in hadronic collisions is important both for accurate tests of the Standard Model and in searches for New Physics. Thanks to the large scale set by the mass of the heavy quark, one can often use QCD perturbation theory in obtaining predictions for heavy-flavor production, employing the factorization of the hadronic cross section into parton distribution functions and perturbative short-distance cross sections.

Top quark production may provide an arena for testing possible extensions of the Standard Model $[1,2,3,4]$. One particularly interesting observable in $t \bar{t}$ production that has been considered in this context $[5,6]$ is the charge asymmetry (or, forward-backward asymmetry), which is obtained by comparing the rate for producing a top quark at a given angle to that for producing an anti-top at the same angle. Electro-weak processes, as well as processes in many extensions of the Standard Model, may produce a charge asymmetry at Born level. QCD, on the other hand, being a purely vector theory, does not produce a charge asymmetry in the lowest-order (LO) processes $q \bar{q} \rightarrow t \bar{t}$ and $g g \rightarrow t \bar{t}$. The charge asymmetry thus has the potential of probing or constraining possible tree-level axial couplings of the gluon $[5,6]$ at the Tevatron or the LHC.

Starting at order $\alpha_{\mathrm{S}}^{3}$, however, QCD itself contributes to the charge asymmetry, through $q \bar{q}$ annihilation $q \bar{q} \rightarrow t \bar{t}(g)$ and flavor excitation, $q g \rightarrow q t \bar{t}[7]$. This happens through diagrams in which two separate fermion lines (one of them the top quark line) are connected by three gluons. This phenomenon, which is also well-known in QED [8], enters with the combination $d_{a b c} d^{a b c}$ of the symmetric QCD structure constants. The same mechanism for light flavors has been found to generate a strange quark $s-\bar{s}$ asymmetry in the nucleon sea [9]. The charge asymmetric part is contained in the full next-to-leading order $\left(\mathrm{NLO}, \mathcal{O}\left(\alpha_{\mathrm{S}}^{3}\right)\right)$ calculations of the differential heavy-flavor production cross section $[10,11,12]$. Since this is the order at which the effect arises for the first time, we will usually refer to the charge asymmetric part arising at $\mathcal{O}\left(\alpha_{\mathrm{S}}^{3}\right)$ as LO. There have been detailed calculations and phenomenological studies of the QCD top quark charge asymmetry at the Tevatron (or the LHC), both for the inclusive case, $p \bar{p} \rightarrow t \bar{t} X[6,13]$, and for associated-jet final states, $p \bar{p} \rightarrow t \bar{t}$ jet $X[14,15]$. In particular, Ref. [15] provides the full NLO $\left(\mathcal{O}\left(\alpha_{\mathrm{S}}^{4}\right)\right)$ corrections to $p \bar{p} \rightarrow t \bar{t}$ jet $X$.

Very recently, first measurements of top quark charge asymmetries have been reported by the Tevatron collaborations $[16,17]$. In the inclusive case, asymmetries of $12 \pm 8$ (stat.) \pm 1 (syst.) $\%$ and $23 \pm 12$ (stat.) \pm 6 (syst.) \% were found by D0 [16] and CDF [17], respectively. Even though experimental uncertainties are evidently still large, this is a very encouraging first step that motivates further theoretical investigations. In the present paper we improve the theoretical framework for the case of the inclusive charge asymmetry by examining the effects of QCD threshold resummation. This will provide insight into the important question of how robust the asymmetry is with respect to higher order QCD corrections. Our study requires us to implement NLL resummation in heavy quark production at fixed angle and rapidity [18, 19]. Earlier phenomenological studies of the threshold-resummed $t \bar{t}$ cross section, which however did not focus on the charge asymmetry, may be found in $[20,21,22,23,24]$.

As is well-known, when the initial partons have just enough energy to produce a $t \bar{t}$ pair, the phase space available for gluon bremsstrahlung nearly vanishes, giving rise to large logarithmic corrections to the partonic cross section. For example, if we consider the cross section for $t \bar{t}$ 
production at fixed pair invariant mass, this partonic threshold is reached when the pair invariant mass equals the partonic center of mass (c.m.) energy, $M_{t \bar{t}}^{2}=s$. At the $n$th order of perturbation theory, the large threshold corrections arise as $\alpha_{\mathrm{S}}^{2+n}\left[\log ^{m}(1-\hat{\tau}) /(1-\hat{\tau})\right]_{+}$with $m \leq 2 n-1$, where $\hat{\tau}=M_{t \bar{t}}^{2} / s$ and the "+"-distribution will be reviewed below. The maximum value, $m=2 n-1$ corresponds to the leading logarithms (LL), $m=2 n-2$ to next-to-leading logarithms (NLL), and so forth. Near threshold, the perturbative calculation produces potentially large corrections at all orders in the strong coupling, $\alpha_{\mathrm{S}}$. These corrections are addressed by threshold resummation. This is particularly relevant for the Tevatron case, where the hadronic c.m. energy is not too much larger than twice the top mass, $2 m_{t}$, so that $\hat{\tau}$ is on average rather close to unity. Related considerations also apply at the LHC when the pair is produced with $M_{t \bar{t}}^{2} \gg 4 m_{t}^{2}$.

For heavy quark production, threshold resummation has been derived to NLL accuracy [21, 18], and recently to next-to-next-to-leading logarithm [24]. The results of [18] have been presented for arbitrary c.m. scattering angle of the produced top quark, which makes it possible to obtain a resummed charge asymmetry from them. Among the processes that contribute to the charge asymmetry, only $q \bar{q}$ annihilation contains threshold logarithms, while the flavor excitation $q g$ process is suppressed near threshold. As we shall discuss in some detail, it turns out that the leading logarithms in the charge asymmetric part of $q \bar{q}$ annihilation cancel at $\mathcal{O}\left(\alpha_{s}^{3}\right)$. This is because the charge asymmetric part is a difference of cross sections with the top or the anti-top produced at a certain angle, and the leading logarithms enter in association with the $\mathcal{O}\left(\alpha_{\mathrm{S}}^{2}\right) q \bar{q} \rightarrow t \bar{t}$ Born process, which is charge symmetric. We shall return to this point below, and will find that beyond $\mathcal{O}\left(\alpha_{\mathrm{S}}^{2}\right)$ the charge asymmetric cross section is enhanced by the same threshold logarithms as the symmetric one. We also note that the $g g$ fusion process is charge symmetric to all orders; nonetheless its resummation can be relevant also for the charge asymmetry as it contributes to the denominator of the asymmetry and may thus dilute it somewhat. This effect does not lead to significant suppression, however, because of the higher-order threshold enhancements to the asymmetric cross section, which we will exhibit below.

The remainder of this paper is organized as follows. In Sec. 2 we give the basic formulas associated with the charge asymmetry and discuss the near-threshold behavior at $\mathcal{O}\left(\alpha_{\mathrm{S}}^{2}\right)$. In Sec. 3 we present the relevant expressions for the NLL resummed $t \bar{t}$ cross section as a function of the $t \bar{t}$ pair invariant mass and the top c.m. scattering angle. Section 4 presents our phenomenological results for Tevatron kinematics, and we summarize our findings in Sec. 5.

\section{Perturbative Cross section, Charge Asymmetry, and Partonic Threshold}

We consider inclusive $t \bar{t}$ production in hadronic collisions,

$$
H_{A}\left(P_{A}\right)+H_{B}\left(P_{B}\right) \rightarrow t\left(p_{t}\right) \bar{t}\left(p_{\bar{t}}\right)+X\left(p_{X}\right)
$$

where we have indicated the momenta. We introduce the invariant mass squared of the $t \bar{t}$ pair: $M_{t \bar{t}}^{2}=\left(p_{t}+p_{\bar{t}}\right)^{2}$, and the variable $\tau \equiv M_{t \bar{t}}^{2} / S$ with $S=\left(P_{A}+P_{B}\right)^{2}$. The factorized cross-section

for the process is written in terms of convolutions of parton distributions $f_{a}^{H_{A}}$ and $f_{b}^{H_{B}}$ for partons 
$a, b$ in hadrons $H_{A}, H_{B}$, respectively, with perturbative partonic hard-scattering cross-sections $\hat{\sigma}_{a b}$ :

$$
\frac{d^{2} \sigma^{H_{A} H_{B} \rightarrow t \bar{t} X}}{d M_{t \bar{t}}^{2} d \cos \theta}=\sum_{a b} \int_{0}^{1} d x_{a} \int_{0}^{1} d x_{b} f_{a}^{H_{A}}\left(x_{a}, \mu^{2}\right) f_{b}^{H_{B}}\left(x_{b}, \mu^{2}\right) \frac{1}{s} \frac{d^{2} \hat{\sigma}_{a b}\left(\hat{\tau}, \cos \theta, \mu^{2} / s\right)}{d \hat{\tau} d \cos \theta},
$$

where the sum runs over all partonic subprocesses that produce top quark pairs. We have introduced the partonic variable $\hat{\tau}=M_{t \bar{t}}^{2} / s=\tau / x_{a} x_{b}$. We define $\theta$ as the production angle of the top quark in the partonic center-of-mass frame. We note from the outset that this is not the definition adopted in the Tevatron experiments, where the asymmetry is considered as a function of the rapidity difference $\Delta y_{t}$ of the $t$ and $\bar{t}$. However, for LO kinematics, the two definitions are directly related through [17] $\tanh (\Delta y / 2)=\beta \cos \theta$, with $\beta=\sqrt{1-4 m_{t}^{2} / s}$ the top quark velocity. As the partonic threshold regime is characterized by LO kinematics, we expect our resummed results below to be very faithful representations also of the effects expected for the Tevatron definition (see also [6]). In fact, we have found that for quantities integrated over angle the charge asymmetries for the two definitions agree at the level of about $2 \%$ or better. The integration limits in Eq. (2) are determined by the conditions [22] $s \geq M_{t \bar{t}}^{2} \geq 4 m_{t}^{2} /\left(1-\beta^{2} \cos ^{2} \theta\right)$. Finally, the scale $\mu$ denotes the factorization and renormalization scales, which we take to be equal throughout this study.

We next define the charge-asymmetric and charge-averaged cross sections:

$$
\begin{aligned}
& \frac{d \Delta \sigma}{d M_{t \bar{t}}^{2} d \cos \theta} \equiv \frac{1}{2}\left\{\frac{d^{2} \sigma^{H_{A} H_{B} \rightarrow t \bar{t} X}}{d M_{t \bar{t}}^{2} d \cos \theta}-\frac{d^{2} \sigma^{H_{A} H_{B} \rightarrow \bar{t} t X}}{d M_{t \bar{t}}^{2} d \cos \theta}\right\}, \\
& \frac{d \bar{\sigma}}{d M_{t \bar{t}}^{2} d \cos \theta} \equiv \frac{1}{2}\left\{\frac{d^{2} \sigma^{H_{A} H_{B} \rightarrow t \bar{t} X}}{d M_{t \bar{t}}^{2} d \cos \theta}+\frac{d^{2} \sigma^{H_{A} H_{B} \rightarrow \bar{t} t X}}{d M_{t \bar{t}}^{2} d \cos \theta}\right\},
\end{aligned}
$$

and the corresponding charge asymmetry

$$
A_{c}\left(M_{t \bar{t}}^{2}, \cos \theta\right) \equiv \frac{d \Delta \sigma}{d \sigma}
$$

To lowest order (LO), $t \bar{t}$ pairs are produced by the processes $q \bar{q} \rightarrow t \bar{t}$ and $g g \rightarrow t \bar{t}$. These produce the top and the anti-top evenly at a given production angle $\theta$, so that the charge asymmetry vanishes. Beyond LO, however, $q \bar{q}$ annihilation as well as the flavor excitation process $q g \rightarrow t \bar{t} q$ have charge asymmetric contributions [13], while $g g$ scattering remains symmetric. For the $q \bar{q}$ annihilation process, the asymmetry arises from three gluons connecting the light-quark and the top-quark lines. In the case of inclusive $t \bar{t}$ production, the asymmetry receives contributions from real diagrams for $q \bar{q} \rightarrow t \bar{t} g$ and from virtual corrections in $q \bar{q} \rightarrow t \bar{t}$. The charge asymmetric pieces for the $\mathcal{O}\left(\alpha_{\mathrm{S}}^{3}\right)$ subprocesses have been computed in detail in [13]; they are also included of course in the full next-to-leading order (NLO) calculations of the top quark cross section $[10,11,12]$.

As we discussed in the Introduction, large double- and single-logarithmic corrections arise at higher orders in the partonic cross sections for $q \bar{q}$ annihilation and $g g$ fusion when $\hat{\tau}$ becomes large, that is, when $M_{t \bar{t}}^{2} \sim s$. The structure of the NLO terms in the $q \bar{q}$ annihilation cross section becomes, for example,

$$
\frac{d^{2} \hat{\sigma}_{q \bar{q}}^{\mathrm{NLO}}(\hat{\tau}, \cos \theta)}{d \hat{\tau} d \cos \theta}=C_{1}(\theta) \delta(1-\hat{\tau})+C_{2}(\theta)\left(\frac{1}{1-\hat{\tau}}\right)_{+}+C_{3}(\theta)\left(\frac{\log (1-\hat{\tau})}{1-\hat{\tau}}\right)_{+}+\ldots
$$


where the ellipses denote terms that are finite near partonic threshold. We have suppressed the dependence on the factorization/renormalization scale, as we will often do in the following. The "+"-distribution is defined as usual by

$$
\int_{x}^{1} d z[g(z)]_{+} f(z)=\int_{x}^{1} d z g(z)(f(z)-f(1))-f(1) \int_{0}^{x} d z g(z) .
$$

The coefficients $C_{i}$ may be found in Ref. [22]. It turns out that only $C_{1}$ and $C_{2}$ possess chargeasymmetric pieces, while the double-logarithmic part associated with $C_{3}$ is symmetric and thus cancels in the asymmetry at lowest order. This is a result of the factorization of collinear logarithms, which do not interfere with color flow in the hard scattering [18]. As a result, the charge asymmetric contributions in $q \bar{q} \rightarrow t \bar{t} g$ and $q \bar{q} \rightarrow t \bar{t}$ each have an infrared (but no collinear) singularity at $\mathcal{O}\left(\alpha_{s}^{3}\right)$ [13], which cancels in their sum and leaves behind a single logarithm, represented by the term proportional to $1 /(1-\hat{\tau})_{+}$in Eq. (4). We will see in the next section, however, that, starting with the next order, leading logarithms contribute to the asymmetric cross section as an overall factor.

\section{NLL Resummation}

The resummation of the soft-gluon contributions is organized in Mellin- $N$ moment space. We take a moment of the hadronic cross section with respect to the variable $\tau$ :

$$
\frac{d \sigma^{N}}{d \cos \theta} \equiv \int_{0}^{1} d \tau \tau^{N-1} \frac{d^{2} \sigma}{d \tau d \cos \theta}
$$

Under Mellin moments, the convolutions in the factorized cross section near threshold become products:

$$
\frac{d \sigma^{N}}{d \cos \theta}=\sum_{a b} f_{a}^{H_{A}, N}\left(\mu^{2}\right) f_{b}^{H_{B}, N}\left(\mu^{2}\right) \hat{\sigma}_{a b}(N, \theta)
$$

where the $f_{a}^{H_{A}, N}, f_{b}^{H_{B}, N}$ are the Mellin moments of the parton distributions, defined by analogy to Eq. (6), and where

$$
\hat{\sigma}_{a b}(N, \theta)=\int_{0}^{1} d \hat{\tau} \hat{\tau}^{N-1} \frac{d^{2} \hat{\sigma}_{a b}(\hat{\tau}, \cos \theta)}{d \hat{\tau} d \cos \theta} .
$$

The threshold limit $(\hat{\tau} \rightarrow 1)$ now corresponds to $N \rightarrow \infty$ in moment space.

Threshold resummation results in exponentiation of the soft gluon corrections in moment space. Unlike color singlet cases like the Drell-Yan process, in heavy-flavor production soft gluons emitted at large angles interfere with the color structure of the underlying Born process. One must then take into account all color structures and sum over them. The details of this procedure were worked out for scattering at fixed angles in $[18,19,25]$. For a given partonic channel $(a b=q \bar{q}$, $\mathrm{gg}$ ), the resumed perturbative cross section is given by ${ }^{\dagger}$

$$
\hat{\sigma}_{a b}^{(\mathrm{res})}(N, \theta)=C_{a b}(\theta) \Delta_{a}(N) \Delta_{b}(N) \operatorname{Tr}\left\{H_{a b}^{(0)}(\theta)\left[\mathcal{S}_{a b}(N, \theta)\right]^{\dagger} S_{a b}^{(0)} \mathcal{S}_{a b}(N, \theta)\right\},
$$

\footnotetext{
${ }^{\dagger}$ See, in particular, Eq. (50) of Ref. [19].
} 
where we have suppressed for simplicity the dependence of the various functions on the pair mass $M_{t \bar{t}}$, but have kept dependence on the scattering angle $\theta$ wherever it occurs. Even before defining the various factors in the resummed cross section, we may note that it naturally gives rise to a charge asymmetry. In Eq. (9), all dependence on the c.m. scattering angle $\theta$ resides in the color trace part and in the coefficients $C_{a b}$. Therefore, these generate the charge-asymmetric part of the cross section near threshold:

$$
\begin{aligned}
\Delta \hat{\sigma}_{q \bar{q}}^{(\mathrm{res})}(N, \theta)= & \left(\Delta_{q}(N)\right)^{2}\left[C_{q \bar{q}}(\theta) \operatorname{Tr}\left\{H_{a b}^{(0)}(\theta)\left[\mathcal{S}_{a b}(N, \theta)\right]^{\dagger} S_{a b}^{(0)} \mathcal{S}_{a b}(N, \theta)\right\}\right. \\
& \left.-C_{q \bar{q}}(-\theta) \operatorname{Tr}\left\{H_{a b}^{(0)}(\theta)\left[\mathcal{S}_{a b}(N,-\theta)\right]^{\dagger} S_{a b}^{(0)} \mathcal{S}_{a b}(N,-\theta)\right\}\right]
\end{aligned}
$$

It is the ratio of Eq. (10) and its charge-averaged counterpart that defines the asymmetry. We anticipate that, when expanding the resummed expression to next-to-leading order, leading logarithms cancel and the $C_{2}$ term in Eq. (4) is reproduced.

Now let us review the elements of the resummed cross sections. The trace in Eq. (9) is taken in a space of color exchange operators $[18,19]$. At lowest order, $H_{a b}^{(0)}$ and $S_{a b}^{(0)}$ are the hard-scattering and the zeroth-order soft functions, respectively. The factors $\mathcal{S}_{a b}$ are also matrices in color space and depend on the basis of color tensors used to describe color exchange. Employing the $s$-channel singlet-octet basis of [18], one has for the $q \bar{q}$ subprocess, which we are mostly interested in here,

$$
\begin{aligned}
H_{q \bar{q}}^{(0)} & =\alpha_{\mathrm{S}}^{2}\left(\begin{array}{cc}
0 & 0 \\
0 & 2\left(\frac{t^{2}+u^{2}}{s^{2}}+2 \frac{m_{t}^{2}}{s}\right) / C_{A}^{2}
\end{array}\right), \\
S_{q \bar{q}}^{(0)} & =\left(\begin{array}{cc}
C_{A}^{2} & 0 \\
0 & \left(C_{A}^{2}-1\right) / 4
\end{array}\right),
\end{aligned}
$$

where $t \equiv\left(p_{t}-p_{a}\right)^{2}-m_{t}^{2}=-s(1-\beta \cos \theta) / 2, u \equiv\left(p_{\bar{t}}-p_{a}\right)^{2}-m_{t}^{2}=-s(1+\beta \cos \theta) / 2$, with $p_{a}$ the momentum of initial parton $a$, again $\beta=\sqrt{1-4 m_{t}^{2} / s}$, and $C_{A}=3$. The corresponding expressions for the soft anomalous dimension matrices of the $g g$-initiated subprocess may be found in [18]. Note that the Born cross sections are recovered by computing $\operatorname{Tr}\left\{H_{a b}^{(0)} S_{a b}^{(0)}\right\}$ and that $H_{a b}^{(0)}$ is symmetric under interchange of $t$ and $u$ and hence charge symmetric.

Each of the functions $\Delta_{a, b}(N)$ and $\mathcal{S}_{a b}(N, \theta)$ is an exponential. $\Delta_{a}(N)$ represents the effects of soft-gluon radiation collinear to initial parton $a$ and in the $\overline{\mathrm{MS}}$ scheme is given by

$$
\ln \Delta_{a}(N)=\int_{0}^{1} \frac{z^{N-1}-1}{1-z} \int_{\mu^{2}}^{(1-z)^{2} M_{t \bar{t}}^{2}} \frac{d q^{2}}{q^{2}} A_{a}\left(\alpha_{\mathrm{S}}\left(q^{2}\right)\right),
$$

and similarly for $\Delta_{b}(N)$. The function $A_{a}$ is a perturbative series in $\alpha_{\mathrm{S}}$,

$$
A_{a}\left(\alpha_{\mathrm{S}}\right)=\frac{\alpha_{\mathrm{S}}}{\pi} A_{a}^{(1)}+\left(\frac{\alpha_{\mathrm{S}}}{\pi}\right)^{2} A_{a}^{(2)}+\ldots
$$

with [26]:

$$
A_{a}^{(1)}=C_{a}, \quad A_{a}^{(2)}=\frac{1}{2} C_{a}\left[C_{A}\left(\frac{67}{18}-\frac{\pi^{2}}{6}\right)-\frac{5}{9} N_{f}\right],
$$

where $N_{f}$ is the number of flavors, and

$$
C_{q}=C_{F}=\left(N_{c}^{2}-1\right) / 2 N_{c}=4 / 3, \quad C_{g}=C_{A}=N_{c}=3 .
$$


The factors $\Delta_{a, b}(N)$ generate leading threshold enhancements, due to soft-collinear radiation, as the same overall factors in the charge symmetric and antisymmetric cross sections.

The large-angle soft gluon exponentials $\mathcal{S}_{a b}(N, \theta)$ are dependent on the process and mix the color structure. One has

$$
\mathcal{S}_{a b}(N, \theta)=\mathcal{P} \exp \left[\frac{1}{2} \int_{M_{t \bar{t}}^{2}}^{M_{t \bar{t}}^{2} / N^{2}} \frac{d q^{2}}{q^{2}} \Gamma_{a b}^{S}\left(\alpha_{\mathrm{S}}\left(q^{2}\right), \theta\right)\right],
$$

where $\mathcal{P}$ denotes path ordering and where $\Gamma_{a b}^{S}$ are soft anomalous dimensions, which are also matrices in a given color basis. They are perturbative; for the resummation at NLL one only needs the first-order term and path ordering becomes irrelevant. For the $q \bar{q}$ subprocess, the firstorder anomalous dimension matrix can be represented, in the $s$-channel singlet-octet basis, as

$$
\Gamma_{q \bar{q}}^{S,(1)}=\frac{\alpha_{\mathrm{S}}}{\pi}\left(\begin{array}{cc}
\Gamma_{11}^{q \bar{q}} & \Gamma_{12}^{q \bar{q}} \\
\Gamma_{21}^{q \bar{q}} & \Gamma_{22}^{q \bar{q}}
\end{array}\right)
$$

with matrix elements $[18]^{\ddagger}$

$$
\begin{aligned}
\Gamma_{11}^{q \bar{q}}= & -C_{F}\left[L_{\beta}+1+i \pi\right] \\
\Gamma_{21}^{q \bar{q}}= & 2 \ln \left(\frac{t}{u}\right) \\
\Gamma_{12}^{q \bar{q}}= & \frac{C_{F}}{C_{A}} \ln \left(\frac{t}{u}\right), \\
\Gamma_{22}^{q \bar{q}}= & C_{F}\left[4 \ln \left(\frac{t}{u}\right)-L_{\beta}-1-i \pi\right] \\
& +\frac{C_{A}}{2}\left[-3 \ln \left(\frac{t}{u}\right)-\ln \left(\frac{m_{t}^{2} s}{t u}\right)+L_{\beta}+1+i \pi\right],
\end{aligned}
$$

where

$$
L_{\beta}=\frac{1-2 m_{t}^{2} / s}{\beta}\left(\ln \frac{1-\beta}{1+\beta}+i \pi\right) .
$$

Finally, the coefficients $C_{a b}(\theta)$ contain $N$-independent hard contributions arising from one-loop virtual corrections. They are perturbative as well, and have the expansion

$$
C_{a b}(\theta)=1+\frac{\alpha_{\mathrm{S}}}{\pi} C_{a b}^{(1)}(\theta)+\mathcal{O}\left(\alpha_{\mathrm{S}}^{2}\right)
$$

The coefficients $C_{a b}^{(1)}$ may be obtained by comparison of the resummed formula to the full NLO calculation. We note that they contain the Coulomb corrections which diverge as $1 / \beta$ at $s \sim 4 m_{t}^{2}$. As indicated, the $C_{a b}^{(1)}$ depend on the scattering angle $\theta$, and in fact for the $q \bar{q}$ subprocess they also contain a charge-asymmetric part. The full coefficients have been derived in Ref. [22] and are given by very lengthy expressions. Starting from slightly corrected versions $\S$ of the expressions given in [22], we have been able to verify that the charge-asymmetric part of the resulting coefficient for the $q \bar{q}$ process reproduces the corresponding result given in [13].

\footnotetext{
${ }^{\ddagger}$ Note that for our definition of the charge asymmetry we need to interchange $t$ and $u$ in the results of [18].

${ }^{\S}$ Specifically, we use Eq. (A.19) of Ref. [27] in the second and third integral in (A.20) of Ref. [28] and in Eqs. (A.9) and (A.11) of the first paper of Ref. [22].
} 
We now give explicit formulas for the expansions of the resummed exponents to NLL accuracy following the general approach of [29]. The functions $\Delta_{a, b}(N)$ become

$$
\ln \Delta_{a}\left(N, \alpha_{\mathrm{S}}\left(\mu^{2}\right), M_{t \bar{t}}^{2} / \mu^{2}\right)=\ln \bar{N} h_{a}^{(1)}(\lambda)+h_{a}^{(2)}\left(\lambda, M_{t \bar{t}}^{2} / \mu^{2}\right)+\mathcal{O}\left(\alpha_{\mathrm{S}}\left(\alpha_{\mathrm{S}} \ln N\right)^{k}\right) .
$$

Here $\lambda=b_{0} \alpha_{\mathrm{S}}\left(\mu^{2}\right) \ln \bar{N}$ with $\bar{N}=N \mathrm{e}^{\gamma_{E}}$, where $\gamma_{E}$ is the Euler constant. The functions $h^{(1,2)}$ are given by

$$
\begin{aligned}
h_{a}^{(1)}(\lambda)= & \frac{A_{a}^{(1)}}{2 \pi b_{0} \lambda}[2 \lambda+(1-2 \lambda) \ln (1-2 \lambda)], \\
h_{a}^{(2)}\left(\lambda, M_{t \bar{t}}^{2} / \mu^{2}\right)= & -\frac{A_{a}^{(2)}}{2 \pi^{2} b_{0}^{2}}[2 \lambda+\ln (1-2 \lambda)]+\frac{A_{a}^{(1)}}{2 \pi b_{0}} \ln (1-2 \lambda) \ln \frac{M_{t \bar{t}}^{2}}{\mu^{2}} \\
& +\frac{A_{a}^{(1)} b_{1}}{2 \pi b_{0}^{3}}\left[2 \lambda+\ln (1-2 \lambda)+\frac{1}{2} \ln ^{2}(1-2 \lambda)\right],
\end{aligned}
$$

where $b_{0}=\left(11 C_{A}-2 N_{f}\right) / 12 \pi$, and

$$
b_{1}=\frac{1}{24 \pi^{2}}\left(17 C_{A}^{2}-5 C_{A} N_{f}-3 C_{F} N_{f}\right) .
$$

The function $h_{a}^{(1)}$ above contains all LL terms in the perturbative series, while $h_{a}^{(2)}$ is of NLL only. For a complete NLL resummation one also needs the expansion of the integral in Eq. (16), given by,

$$
\ln \mathcal{S}_{a b}(N, \theta)=\frac{\ln (1-2 \lambda)}{2 \pi b_{0}} \Gamma_{q \bar{q}}^{S,(1)}(\theta)
$$

While the full structure of the resummed expressions is rather long and complicated, a major simplification occurs when one expands it for small $\beta$ and ignores the coefficient $C_{a b}$. One finds in this case

$$
\hat{\sigma}_{q \bar{q}}^{(\mathrm{res})}(N, \theta)=\hat{\sigma}_{q \bar{q}}^{(\mathrm{Born})}(\theta)\left(\Delta_{q}(N)\right)^{2}\left\{1+\frac{\beta \cos \theta\left(8 C_{F}-3 C_{A}\right) \ln (1-2 \lambda)}{\pi b_{0}}\right\} \mathrm{e}^{-\frac{C_{A}}{2 \pi b_{0}} \ln (1-2 \lambda)} .
$$

Here, the factor $8 C_{F}-3 C_{A}$ is the typical color factor associated with the QCD charge asymmetry [13]. One can see how the single threshold logarithm arises at the first order in $\alpha_{\mathrm{S}}$ in the charge-asymmetric part. The charge-asymmetric piece is suppressed by the factor $\beta$, but enhanced by the term $\ln (1-2 \lambda)$. All factors outside the curly brackets are common to the charge-asymmetric and the charge-summed parts and are expected to largely cancel in the charge asymmetry at hadron level. We note that in the limit $\beta \rightarrow 0$ our formulas above reproduce the moment-space expressions for the resummed total heavy-flavor cross section derived in [21].

In our discussion below, we use the full formula (10) when calculating the charge asymmetry. Since the matrices involved for the $q \bar{q}$ subprocess are two-dimensional, it is straightforward to perform the required exponentiations and other manipulations, explicitly employing a diagonal color basis for these $2 \times 2$ matrices [18]. For the (charge-symmetric) $g g$ subprocess, the matrices are three-dimensional, and this procedure becomes more complicated. We found it simpler here to do the matrix manipulations numerically, calculating in particular the matrix exponentials by expanding the exponential series to the tenth order. We emphasize again that the $g g$ process is charge-symmetric. Thus, it only contributes to the denominator of the charge asymmetry, diluting the asymmetry somewhat, because the effects of threshold resummation can be larger for gluons than for quarks. 


\section{Phenomenological Results}

We will now investigate the numerical size of the QCD charge asymmetry for top quark production at the Tevatron, making use of the resummation formulas presented above. In order to do this, we first need to specify the inverse Mellin transform. This requires a prescription for dealing with the singularity in the perturbative strong coupling constant in the resummed exponent. We will use the Minimal Prescription developed in Ref. [29], which relies on use of the NLL expanded forms Eqs. (21)-(23), and on choosing a Mellin contour in complex- $N$ space that lies to the left of the poles at $\lambda=1 / 2$ in the Mellin integrand:

$$
\frac{d^{2} \sigma}{d M_{t \bar{t}}^{2} d \cos \theta}=\int_{C_{M P}-i \infty}^{C_{M P}+i \infty} \frac{d N}{2 \pi i} \tau^{-N} \sigma^{(\mathrm{res})}(N),
$$

where $b_{0} \alpha_{\mathrm{S}}\left(\mu^{2}\right) \ln C_{M P}<1 / 2$, but all other poles in the integrand are as usual to the left of the contour. The result defined by the minimal prescription has the property that its perturbative expansion is an asymptotic series that has no factorial divergence and therefore no "built-in" power-like ambiguities. Power corrections may then be added, as phenomenologically required.

For our calculations we use the CTEQ6M parton distributions [30]. To obtain these in Mellinmoment space, we follow [31] and perform a simple fit to each parton distribution at each scale needed, using a functional form that allows Mellin moments to be taken analytically. We note that for the Tevatron case considered here, $t \bar{t}$ pairs are largely produced in valence-valence scattering, for which the parton distributions are rather well known. The higher-order and resummation effects will be very similar for other sets of parton distributions. We use $m_{t}=170.9 \mathrm{GeV}$ [32], $\sqrt{S}=1.96 \mathrm{TeV}$, and our default choice for the factorization/renormalization scale is $\mu=M_{t \bar{t}}$.

The lower set of lines in Fig. 1 shows our results for the charge asymmetric cross section $d \Delta \sigma / d M_{t \bar{t}}$ as a function of the $t \bar{t}$ pair mass, while the upper set presents the charge-averaged one, $d \bar{\sigma} / d M_{t \bar{t}}$. We show here the cross sections integrated over $0 \leq \cos \theta \leq 1$. In both cases, we show by the dotted lines the lowest-order result, which is $\mathcal{O}\left(\alpha_{\mathrm{S}}^{2}\right)$ in the charge-averaged case, and $\mathcal{O}\left(\alpha_{\mathrm{S}}^{3}\right)$ in the asymmetric part. In the former case, this result is just based on the usual Born cross processes, $q \bar{q} \rightarrow t \bar{t}$ and $g g \rightarrow t \bar{t}$. For the charge asymmetric part, we make use of the expressions given in the Appendix of [13]. This includes the small contribution by the quark-gluon flavor excitation process $q g \rightarrow t \bar{t} q$. Next in Fig. 1 we show the first-order expansion of the resummed cross sections (dashed lines), which are approximations to the full NLO result. In case of the charge-asymmetric piece, which only starts at NLO, we can check the quality of this approximation by comparing the dotted and dashed lines. One can see that the two results agree very well in this case, implying that the threshold corrections addressed by resummation dominate. 9

For the charge-asymmetric part, we also show (dashed-dotted lines) the second-order expansion of the resummed cross section, which is of $\mathcal{O}\left(\alpha_{\mathrm{S}}^{4}\right)$, because this contains the first non-trivial QCD correction in this case. The solid lines finally show the full NLL-resummed result. One can see that resummation has a very significant impact on the predicted cross sections, in particular for the charge-asymmetric part at high $M_{t \bar{t}}$. Note that for the resummed curve for this part we have performed a matching to the full $\mathcal{O}\left(\alpha_{\mathrm{S}}^{3}\right)$ result of [13] by correcting it by the difference between the

๑In principle, one may carry out the same check for the charge-symmetric cross section at fixed $M_{t \bar{t}}$ and $\theta$ by comparing to the full NLO calculations of $[10,11]$. Such a study is unlikely to change our results here qualitatively, and would go beyond the scope of this work. 
dotted and dashed lines. In this way, the $\mathcal{O}\left(\alpha_{\mathrm{S}}^{3}\right)$ is taken into account in full, and the soft-gluon contributions beyond NLO are resummed to NLL.

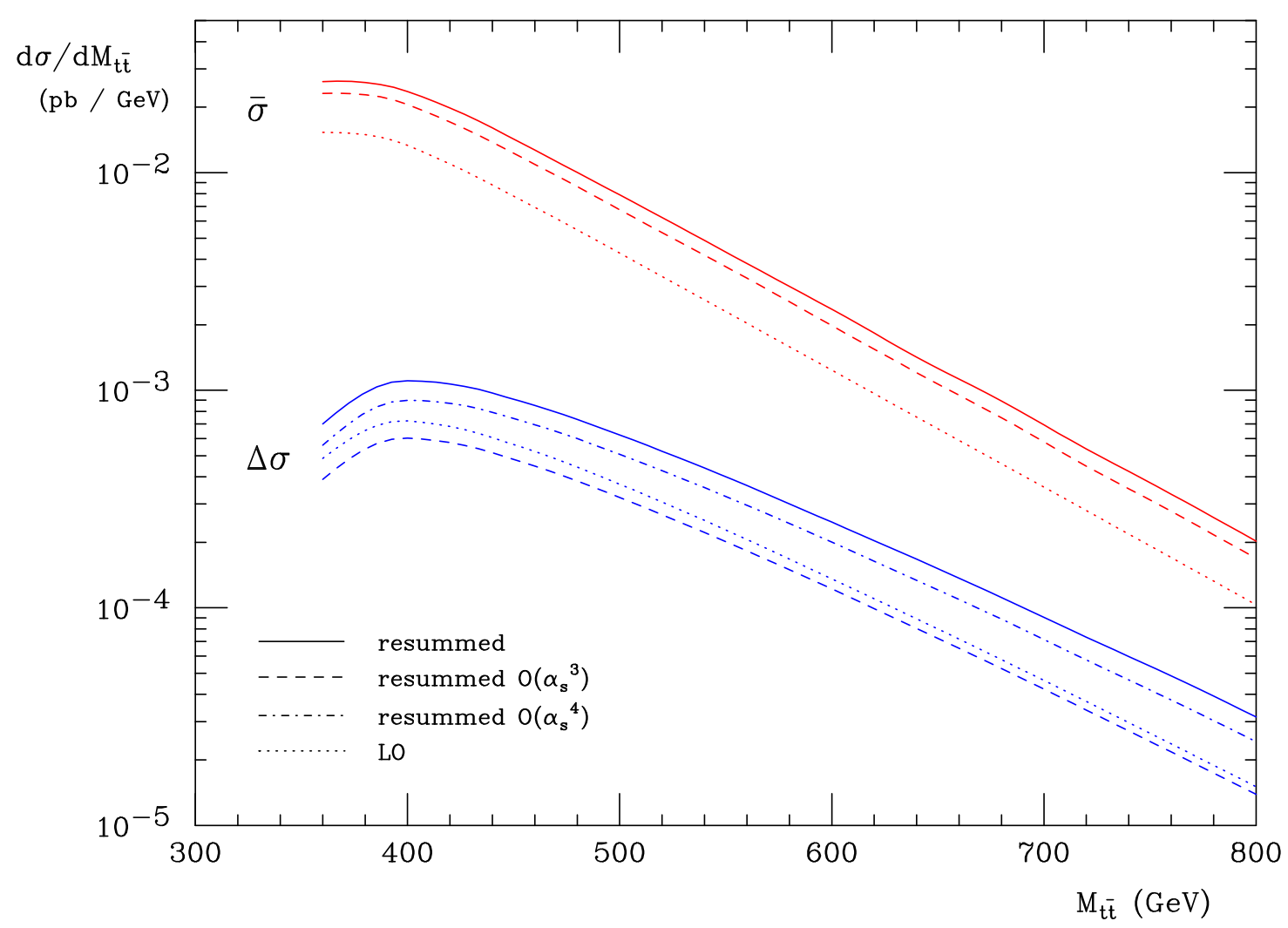

Figure 1: Charge asymmetric and charge averaged cross sections $d \Delta \sigma / d M_{t \bar{t}}$ and $d \bar{\sigma} / d M_{t \bar{t}}$ as functions of the $t \bar{t}$ pair mass, integrated over $0 \leq \cos \theta \leq 1$. Dotted lines are LO, dashed lines include the first-order corrections generated by resummation, and solid lines show the full resummed result. For the charge-asymmetric part, we also show the second-order expansion of the resummed cross section, which is of $\mathcal{O}\left(\alpha_{\mathrm{S}}^{4}\right)$, because this contains the first non-trivial QCD correction in this case.

In Fig. 2 we analyze the scale dependence of the results, for both the charge asymmetric and averaged parts. The dash-dotted lines correspond to a variation of $M_{t \bar{t}} / 2 \leq \mu \leq 2 M_{t \bar{t}}$, with the central lines (dotted) the ones for $\mu=M_{t \bar{t}}$ already shown in Fig. 1. The higher results are obtained for the lower choice of scale. The dashed and solid lines display the same for the resummed cross sections. One can see a very significant reduction of scale dependence, in particular for the charge averaged cross section. This improvement in scale dependence due to threshold resummation is in line with similar findings in the literature for other cross sections [21, 22, 23, 33, 34]. We note that the fact that $t \bar{t}$ production at the Tevatron proceeds primarily through $q \bar{q}$ valence-valence annihilation helps here, since only the flavor-diagonal non-singlet evolution matters, which is part of the resummation formula $[21,33]$.

Figure 3 shows the charge asymmetries $A_{c}=d \Delta \sigma / d \bar{\sigma}$ corresponding to the various curves in Fig. 1, as functions of the pair mass. As before, the dotted line shows the LO result, the solid represents the full resummed result, and the dashed one is the expansion of the resummed cross section. For the latter we expand the cross section to $\mathcal{O}\left(\alpha_{\mathrm{S}}^{4}\right)$ in the numerator of the asymmetry, 


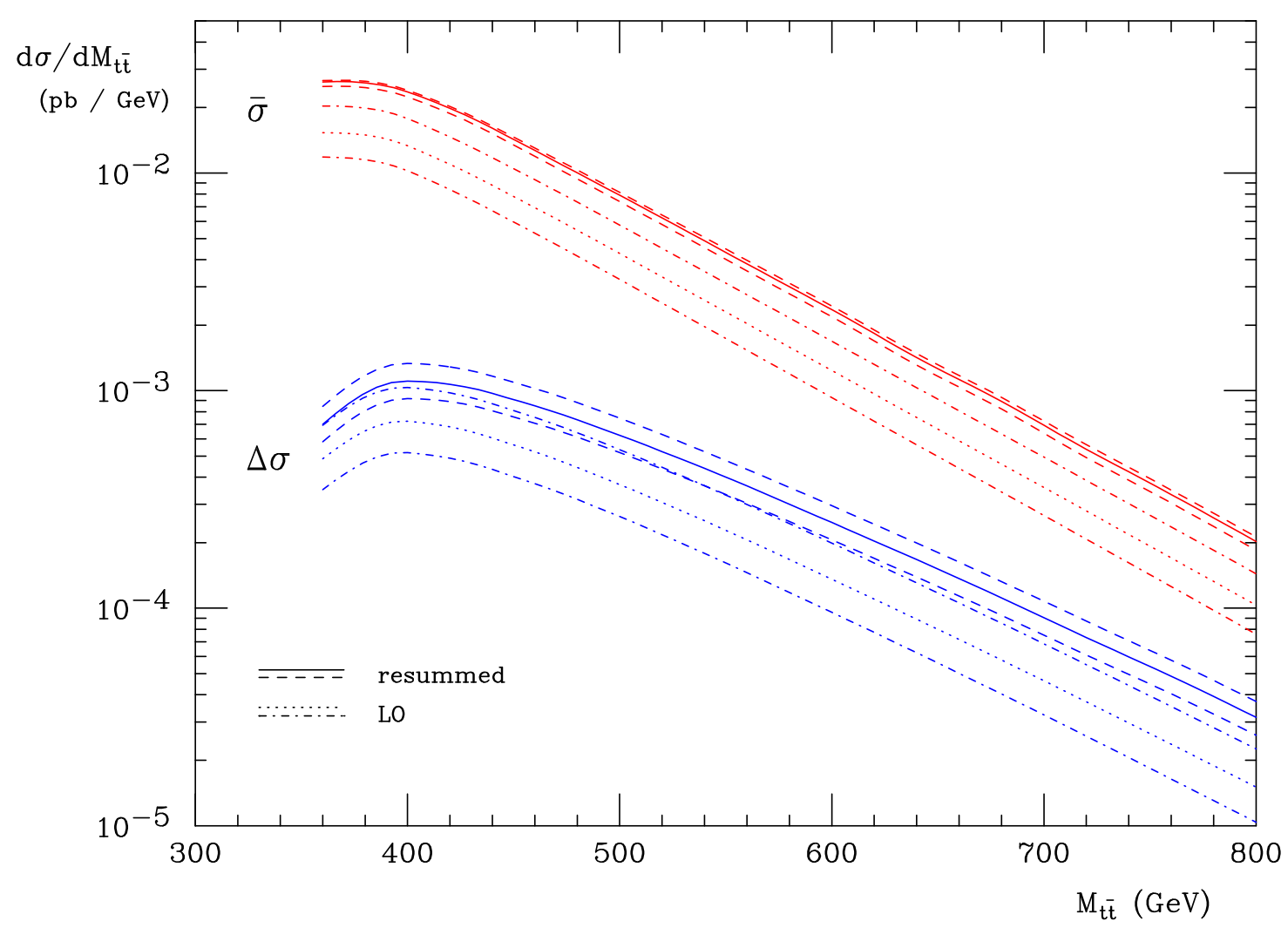

Figure 2: Scale dependence of the LO (dotted and dash-dotted) and NLL resummed (solid and dashed) cross sections, for a scale variation $M_{t \bar{t}} / 2 \leq \mu \leq 2 M_{t \bar{t}}$.

and to $\mathcal{O}\left(\alpha_{\mathrm{S}}^{3}\right)$ in the denominator, thus taking into account the first non-trivial QCD correction in both cases. Had we expanded both numerator and denominator to $\mathcal{O}\left(\alpha_{\mathrm{S}}^{3}\right)$, the numerator would be at LO, and an artificially small asymmetry would result. One can see that the various results are rather close, implying that the net effect of resummation on the charge asymmetry is not large. This is related to the fact that the double-logarithmic factors $\propto\left(\Delta_{q}(N)\right)^{2}$ in the resummation formula are the same for the charge asymmetric and averaged parts. Towards lower $M_{t \bar{t}}$, where the speed of the produced top quark becomes small, all asymmetries become small on account of Eq. (26). At large pair masses, the additional single threshold logarithm enhances the asymmetry.

In Fig. 4 we consider the asymmetry as a function of $\cos \theta$, with $M_{t \bar{t}}$ integrated over the allowed kinematic region. Again the net effect of resummation on the asymmetry is relatively moderate. Integration over $M_{t \bar{t}}$ leads generally to a smaller asymmetry, because lower pair masses, at which the asymmetry decreases when going from the LO to the resummed case (see Fig. 3), dominate the cross section. As Figs. 3 and 4 show, the resummed asymmetries grow substantially with both pair mass, when integrated over rapidity, and with the relative rapidity of the pair. Interestingly, these results are consistent with the explicit NLO results presented in Refs. [14, 15], which indicate a decrease in the charge asymmetry, and even a reversal of its sign, for top pair plus jet cross sections. In such final states, the NLO virtual corrections to inclusive pair production are absent, and it is the latter corrections that determine the sign of the asymmetry itself.

We finally turn to the total charge asymmetry $A_{c}^{\text {tot. }}$, integrated over $M_{t \bar{t}}$ and $0 \leq \cos \theta \leq 1$. At LO, using the scale $\mu=M_{t \bar{t}} / 2$, we find $A_{c}^{\text {tot. }}=6.7 \%$. Resummation results in only a small change, $A_{c}^{\text {tot. }}=6.6 \%$. We note that when varying the scale over the range $m_{t} \leq \mu \leq M_{t \bar{t}}$, the 


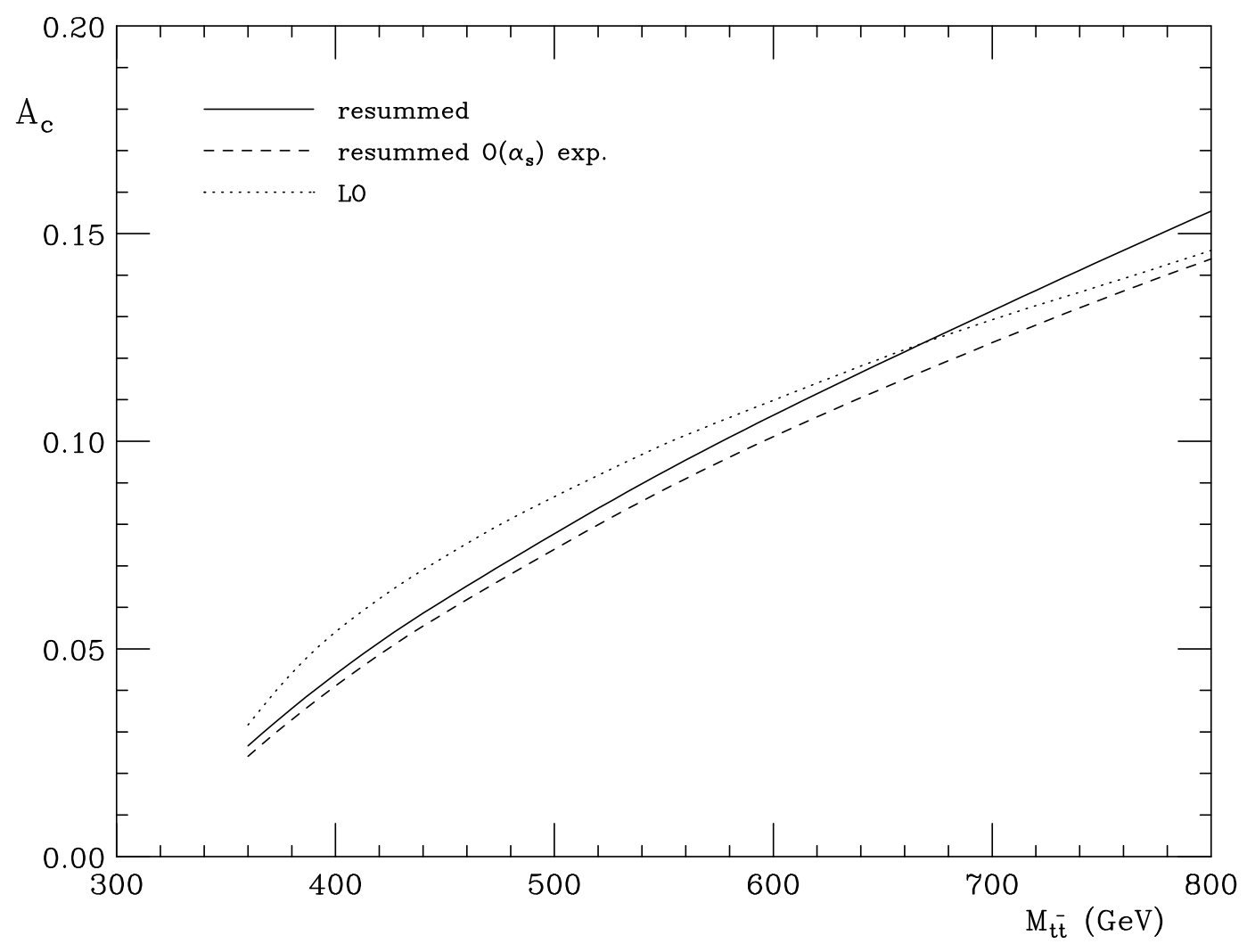

Figure 3: Charge asymmetry corresponding to the curves in Fig. 1.

LO charge-symmetric part of the cross section varies by about $\pm 20 \%$ around its central value, which is improved by resummation to a variation of about $\pm 3 \%$. The scale dependence of the asymmetric part of the cross section improves from $\pm 28 \%$ to $\pm 13 \%$. The resummed asymmetry shows a variation over this range of scales of about $\pm 12 \%$. Thus our results for the higher-order corrections to $A_{c}^{\text {tot. }}$ are well consistent with the estimate of a $\sim 30 \%$ uncertainty made in Ref. [6].

\section{Conclusions and Outlook}

We have presented a study of the next-to-leading logarithmic QCD threshold resummation effects on the charge asymmetry in inclusive $t \bar{t}$ production at the Tevatron. We have found that the asymmetry is stable with respect to the higher-order corrections generated by threshold resummation. We have also found that resummation significantly decreases the dependence of the results on the factorization and renormalization scales, thus making the Standard Model prediction for the asymmetry more reliable.

It will be interesting to extend these studies to the case of $t \bar{t}$ jet production, for which sizable negative NLO corrections have been found [15]. Also, there will be interesting applications at the LHC in situations near partonic threshold, i.e., when the $t \bar{t}$ pair mass becomes of the order of $1 \mathrm{TeV}$ or larger. Because the initial $p p$ state is symmetric, one needs to apply additional cuts (for example, on the $t \bar{t}$ pair rapidity) here in order to generate a non-vanishing charge asymmetry [13]. 


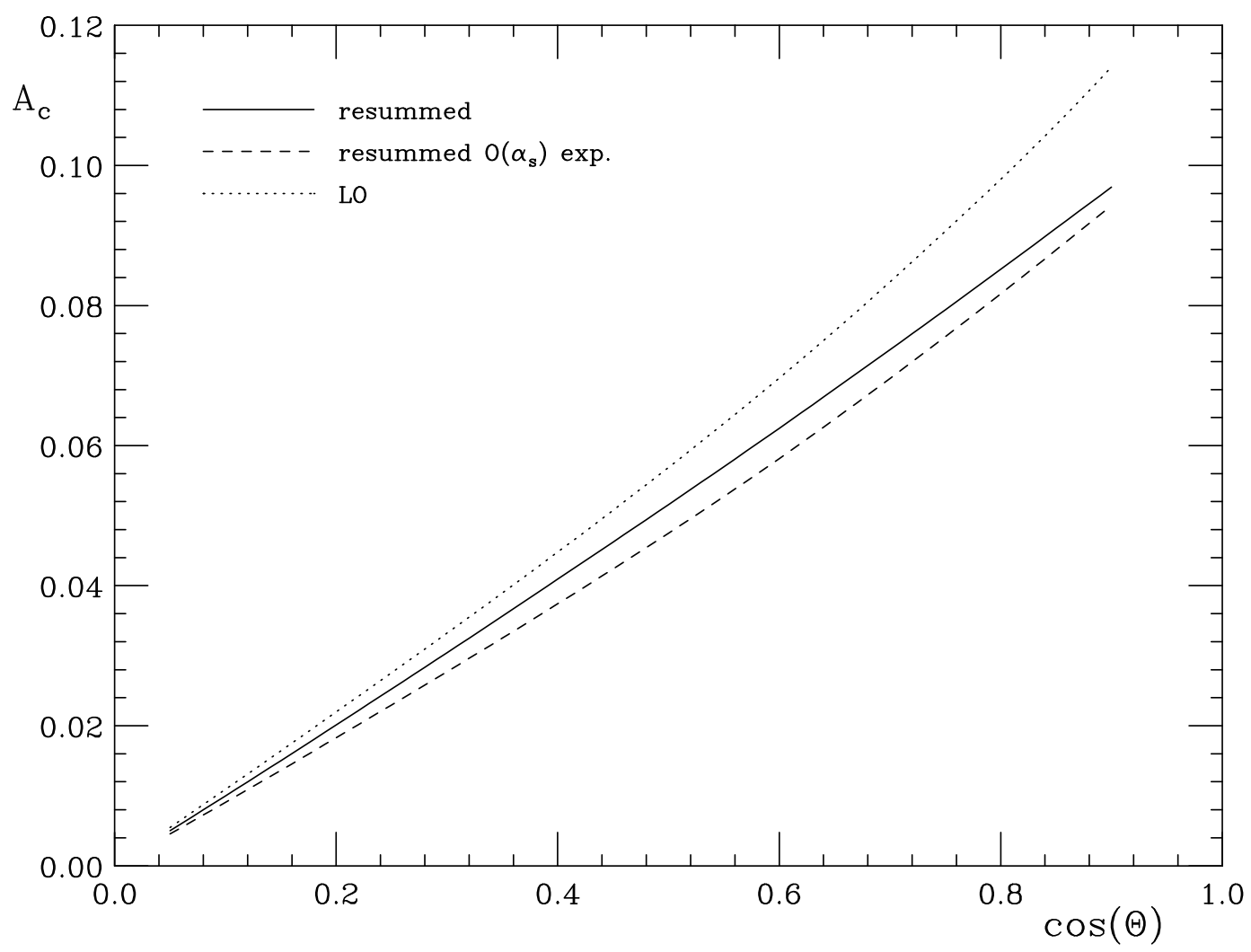

Figure 4: Same as Fig. 3, but as a function of $\cos \theta$, integrated over the $t \bar{t}$ pair mass.

\section{Acknowledgments}

We are grateful to E. Laenen for very helpful communications and to D. de Florian, G. Perez, G. Rodrigo, J. Smith, and M. Stratmann for useful discussions. W.V. is grateful to the U.S. Department of Energy (contract number DE-AC02-98CH10886) for providing the facilities essential for the completion of his work. This work was supported in part by the National Science Foundation, grants PHY-0354776, PHY-0354822 and PHY-0653342.

\section{References}

[1] K. D. Lane, Phys. Rev. D 52, 1546 (1995) [arXiv:hep-ph/9501260].

[2] For recent work, see contribution of D.G.E. Walker in G. Brooijmans et al., "New Physics at the LHC: A Les Houches Report. Physics at Tev Colliders 2007 - New Physics Working Group," arXiv:0802.3715 [hep-ph].

[3] D. Atwood, S. Bar-Shalom, G. Eilam and A. Soni, Phys. Rept. 347, 1 (2001) [arXiv:hep$\mathrm{ph} / 0006032]$.

[4] For review, see: W. Bernreuther, arXiv:0805.1333 [hep-ph].

[5] L. M. Sehgal and M. Wanninger, Phys. Lett. B 200, 211 (1988); J. Bagger, C. Schmidt and S. King, Phys. Rev. D 37, 1188 (1988). 
[6] O. Antuñano, J. H. Kühn and G. V. Rodrigo, Phys. Rev. D 77, 014003 (2008) [arXiv:0709.1652 [hep-ph]]; G. Rodrigo, arXiv:0803.2992 [hep-ph].

[7] F. Halzen, P. Hoyer and C. S. Kim, Phys. Lett. B 195, 74 (1987).

[8] R. W. Brown, K. O. Mikaelian, V. K. Cung and E. A. Paschos, Phys. Lett. B 43, 403 (1973); F. A. Berends, K. J. F. Gaemers and R. Gastmans, Nucl. Phys. B 63, 381 (1973); S. J. Brodsky, C. E. Carlson and R. Suaya, Phys. Rev. D 14, 2264 (1976).

[9] S. Catani, D. de Florian, G. Rodrigo and W. Vogelsang, Phys. Rev. Lett. 93, 152003 (2004) [arXiv:hep-ph/0404240].

[10] W. Beenakker, H. Kuijf, W. L. van Neerven and J. Smith, Phys. Rev. D 40, 54 (1989); W. Beenakker, W. L. van Neerven, R. Meng, G. A. Schuler and J. Smith, Nucl. Phys. B 351, 507 (1991).

[11] P. Nason, S. Dawson and R. K. Ellis, Nucl. Phys. B 327, 49 (1989) [Erratum-ibid. B 335, $260(1990)]$.

[12] J. Riedl, A. Schäfer and M. Stratmann, Eur. Phys. J. C 52, 987 (2007) [arXiv:0708.3010 [hep-ph]].

[13] J. H. Kühn and G. Rodrigo, Phys. Rev. Lett. 81, 49 (1998) [arXiv:hep-ph/9802268]; Phys. Rev. D 59, 054017 (1999) [arXiv:hep-ph/9807420].

[14] M. T. Bowen, S. D. Ellis and D. Rainwater, Phys. Rev. D 73, 014008 (2006) [arXiv:hep$\mathrm{ph} / 0509267]$.

[15] S. Dittmaier, P. Uwer and S. Weinzierl, Phys. Rev. Lett. 98, 262002 (2007) [arXiv:hep$\mathrm{ph} / 0703120]$.

[16] V. M. Abazov et al. [D0 Collaboration], arXiv:0712.0851 [hep-ex].

[17] J. Weinelt, Masters thesis, Universität Karlsruhe, FERMILAB-MASTERS-2006-05; IEKPKA-2006-21; D. Hirschbuehl, Ph.D. Thesis, Universität Karlsruhe, FERMILAB-THESIS$2005-80$.

[18] N. Kidonakis and G. Sterman, Nucl. Phys. B 505, 321 (1997) [arXiv:hep-ph/9705234].

[19] N. Kidonakis, G. Oderda and G. Sterman, Nucl. Phys. B 525, 299 (1998) [arXiv:hep$\mathrm{ph} / 9801268]$.

[20] E. Laenen, J. Smith and W. L. van Neerven, Phys. Lett. B 321, 254 (1994) [arXiv:hepph/9310233]; S. Catani, M. L. Mangano, P. Nason and L. Trentadue, Phys. Lett. B 378, 329 (1996) [arXiv:hep-ph/9602208]; E. L. Berger and H. Contopanagos, Phys. Rev. D 54, 3085 (1996) [arXiv:hep-ph/9603326].

[21] R. Bonciani, S. Catani, M. L. Mangano and P. Nason, Nucl. Phys. B 529, 424 (1998) [arXiv:hep-ph/9801375].

[22] N. Kidonakis, E. Laenen, S. Moch and R. Vogt, Phys. Rev. D 64, 114001 (2001) [arXiv:hepph/0105041]; N. Kidonakis and R. Vogt, Phys. Rev. D 68, 114014 (2003) [arXiv:hep$\mathrm{ph} / 0308222]$. 
[23] M. Cacciari, S. Frixione, M. L. Mangano, P. Nason and G. Ridolfi, arXiv:0804.2800 [hep-ph].

[24] S. Moch and P. Uwer, arXiv:0804.1476 [hep-ph].

[25] R. Bonciani, S. Catani, M. L. Mangano and P. Nason, Phys. Lett. B 575, 268 (2003) [arXiv:hep-ph/0307035].

[26] J. Kodaira and L. Trentadue, Phys. Lett. B 112, 66 (1982); Phys. Lett. B 123, 335 (1983);

S. Catani, E. D'Emilio and L. Trentadue, Phys. Lett. B 211, 335 (1988).

[27] S. Frixione, M. L. Mangano, P. Nason and G. Ridolfi, Nucl. Phys. B 412, 225 (1994) [arXiv:hep-ph/9306337].

[28] M. L. Mangano, P. Nason and G. Ridolfi, Nucl. Phys. B 373, 295 (1992).

[29] S. Catani, M. L. Mangano, P. Nason and L. Trentadue, Nucl. Phys. B 478, 273 (1996) [arXiv:hep-ph/9604351].

[30] J. Pumplin, D. R. Stump, J. Huston, H. L. Lai, P. Nadolsky and W. K. Tung, JHEP 0207, 012 (2002) [arXiv:hep-ph/0201195].

[31] G. Bozzi, S. Catani, D. de Florian and M. Grazzini, Phys. Lett. B 564, 65 (2003) [arXiv:hepph/0302104]; D. de Florian and J. Zurita, Phys. Lett. B 659, 813 (2008) [arXiv:0711.1916 [hep-ph]].

[32] CDF and D0 Collaborations, arXiv:hep-ex/0703034.

[33] G. Sterman and W. Vogelsang, in: High Energy Physics 99, Proceedings of the "International Europhysics Conference on High-Energy Physics", ed. K. Huitu et al. (Institute of Physics Publishing, Bristol, UK, 2000), hep-ph/9910371.

[34] see, for example: S. Catani, M. L. Mangano, P. Nason, C. Oleari and W. Vogelsang, JHEP 9903, 025 (1999) [arXiv:hep-ph/9903436]; N. Kidonakis and J. F. Owens, Phys. Rev. D 61, 094004 (2000) [arXiv:hep-ph/9912388]; G. Sterman and W. Vogelsang, JHEP 0102, 016 (2001) [arXiv:hep-ph/0011289]; S. Catani, D. de Florian, M. Grazzini and P. Nason, JHEP 0307, 028 (2003) [arXiv:hep-ph/0306211]; D. de Florian and W. Vogelsang, Phys. Rev. D 72, 014014 (2005) [arXiv:hep-ph/0506150]; Phys. Rev. D 71, 114004 (2005) [arXiv:hep$\mathrm{ph} / 0501258]$. 